\title{
Defects in mitochondrial RNA metabolism in disease
}

Stefan J. Siira ${ }^{1}$, Anne-Marie J. Shearwood ${ }^{1}$, Cameron P. Bracken ${ }^{2}$, Oliver Rackham ${ }^{1,3}$ and Aleksandra Filipovska ${ }^{1,3} *$

${ }^{1}$ Harry Perkins Institute of Medical Research and Centre for Medical Research, Level 7 QQ Block, QEII Medical Centre, 6 Verdun Street, Nedlands WA 6009 Australia;

2 Division of Human Immunology, Centre for Cancer Biology, SA Pathology, Adelaide SA 5000, Australia

3 School of Chemistry and Biochemistry, The University of Western Australia, Nedlands, Western Australia 6009, Australia.

* Correspondence: aleksandra.filipovska@uwa.edu.au; Tel.: +61-8-6151-0732 


\begin{abstract}
The expression of mitochondrially-encoded genes requires the efficient processing of long precursor RNAs at the 5' and 3' ends of tRNAs, a process which, when disrupted, results in disease. Two such mutations reside within $m t-t R N A^{\operatorname{Leu}(U U R)}$; a m.3243A $>\mathrm{G}$ transition, which is the most common cause of MELAS (mitochondrial myopathy, encephalopathy, lactic acidosis and strokelike episodes), and m.3302A $>\mathrm{G}$ which often causes mitochondrial myopathy (MM). We used parallel analysis of RNA ends (PARE) that captures the $5^{\prime}$ terminal end of $5^{\prime}$-monophosphorylated mitochondrial RNAs to compare the effects of the m.3243A>G and m.3302A $>\mathrm{G}$ mutations on mitochondrial tRNA processing and downstream RNA metabolism. We confirmed previously identified RNA processing defects, identified common internal cleavage sites and new sites unique to the m.3243A $>\mathrm{G}$ mutants that do not correspond to transcript ends. These sites occur in regions of predicted RNA secondary structure, or are in close proximity to such regions, and may identify regions of importance to the processing of mtRNAs.
\end{abstract}

Key words: RNA metabolism; mitochondrial disease; tRNA; PARE 


\section{Introduction}

Mitochondria contain their own discrete genomes, a characteristic that is borne from their $\alpha$ proteobacterial origin. The human mitochondrial genome is a circular molecule 16,569 bp in length that encodes 13 protein components of the electron transport chain, 2 mitochondrial rRNAs and 22 mitochondrial tRNAs. Mitochondrial RNAs are initially transcribed as long, polycistronic transcripts from both strands of the genome denoted the heavy and light strands due to their high and low purine content, respectively, which are processed to excise interspersed tRNAs thus releasing the other transcripts (reviewed in (Rackham et al., 2012)). Both strands are transcribed from promoters located within the non-coding region containing the D-loop, known as the control region, with light strand transcript termination occurring at the MTERF1 site within the MT-TL1 mtDNA region while the heavy-strand transcript is almost genome-length and terminates upstream of the D-loop (reviewed in (Gustafsson et al., 2016)). Following transcription of mitochondrial DNA (mtDNA), the $5^{\prime}$ and $3^{\prime}$ ends of tRNAs residing within the precursor transcripts are cleaved by the mitochondrial RNase P and RNase Z (ELAC2) (Brzezniak et al., 2011; Holzmann et al., 2008; Rackham et al., 2016; Sanchez et al., 2011), respectively to release individual rRNAs, mRNAs and tRNAs. These mitochondrial RNAs (mtRNAs) undergo maturation processes involving various post-transcriptional modifications such as nucleotide modifications of tRNAs and rRNAs, 3' CCA trinucleotide additions to tRNAs and polyadenylation of mRNAs (reviewed in (Rackham et al., 2012)). Degradation of mitochondrial RNAs is mediated by the hSUV3-hPNPase complex in a process that appears to be functionally coupled with polyadenylation, although many aspects of mitochondrial degradation remain unknown (reviewed in (Levy and Schuster, 2016)).

In the mitochondrial genome, tRNA genes have been noted as hotspots for pathological mutations and MT-TL1 in particular (Moraes et al., 1993), perhaps due to its dual importance in the processing of rRNA-containing precursor RNA and as the location of an MTERF1-binding site that mediates transcriptional termination. An A to $G$ transition at nucleotide position 3243, which resides within MT-TL1, is one of the most studied mitochondrial mutations due to its frequency and association with a number of mitochondrial diseases, most notably mitochondrial myopathy, encephalomyopthy, lactic acidosis and stroke-like episodes (MELAS) where it is responsible for $\sim 80 \%$ of cases, but also myoclonus epilepsy with ragged-red fibres (MERRF), mitochondrial myopathy (MM), Leigh syndrome (LS), chronic progressive external opthalmoplgeia (CPEO) and maternally inherited diabetes and deafness (MIDD) (reviewed in (Kirino and Suzuki, 2005)). High load of this mutation results in a decreased rate of protein synthesis, compromised cellular respiration and reduced steady-state levels of mitochondrially-encoded respiratory chain components (King et al., 1992). 
At a molecular level, the mutation has been shown to have multiple effects on the tRNA itself including impaired post-transcriptional modification such as aminoacylation (Börner et al., 2000), methylation (Helm et al., 1999) and taurine modification of the anticodon wobble base (Kirino et al., 2004), alterations to its tertiary structure leading to dimerization (Wittenhagen and Kelley, 2002), and misincorporation of amino acids in elongating peptides leading to elevated degradation (Sasarman et al., 2008). Furthermore, reduced efficiency of 5' and 3'MT-TL1 cleavage disrupts the processing of mitochondrial precursor RNA, as is evident by the accumulation of an intermediate RNA product known as RNA19 (Hess et al., 1991), that contains the MT-RNR2, MTTL1 and MT-ND1 transcripts, observed both in patient muscle and transmitochondrial cybrids close to homoplasmy (King et al., 1992; Maniura-Weber et al., 2006).

The accumulation of RNA19 as a consequence of another mutation at position m.3302A>G in MT-TL1 has been associated most often with mitochondrial myopathy (Bindoff et al., 1993), but has also been linked to MELAS (Goto et al., 2014) as well as other disorders (van den Bosch et al., 2004). The mutation of this highly conserved nucleotide impairs $3^{\prime}$ processing of MT-TL1 (Levinger et al., 2004) leading to a reduction in steady-state levels and partially impaired aminoacylation, the accumulation of stable RNA19 (Maniura-Weber et al., 2006) and a respiratory defect that predominantly affects complex I (Bindoff et al., 1993).

Parallel analysis of RNA ends (PARE) is an approach that was developed to investigate RNAs with a monophosphate at the 5' end such as those found in miRNAs, RNA degradation products (German et al., 2009) and mitochondrial RNAs (Mercer et al., 2011; Rackham et al., 2016). The 5' monophoshpate of cleaved RNAs allows the ligation of an adapter containing a MmeI recognition site, which after reverse transcription and cDNA synthesis the restriction enzyme MmeI is used to cleave the cDNA $20 \mathrm{nt}$ downstream of its recognition site to fragments that are amplified and sequenced. Mitochondrial transcripts possess 5' terminal monophosphates (Rackham et al., 2016), in contrast to the 5' $\mathrm{m} 3 \mathrm{G}$ protective caps present on nuclear mRNAs, making PARE amenable for investigation of RNA processing in mitochondria. Analysis of the abundance and distribution of the terminal 5' nucleotide of these tags produces a profile of both specific and nonspecific cleavage events, such as degradation, which appear as large single nucleotide peaks or as series of stepped peaks in close proximity, respectively (Rackham et al., 2016).

In this study, we used PARE to investigate the effects of the m.3243A>G and m.3302A>G mutations on mitochondrial RNAs and identify any transcriptome-wide differences in their processing or degradation as a consequence.

\section{Materials and Methods}




\subsection{Cell culture}

Cybrid cell lines were cultured at $37{ }^{\circ} \mathrm{C}$ under humidified 95\% air/5\% CO2 in Dulbecco's modified Eagle's medium (DMEM, Gibco, Life Technologies) containing glucose (4.5 g/l- 1), 1 $\mathrm{mM}$ pyruvate, $50 \mu \mathrm{g} / \mathrm{ml}$ uridine, $2 \mathrm{mM}$ glutamine, penicillin $(100 \mathrm{U} / \mathrm{ml}-1)$, streptomycin sulfate $(100 \mu \mathrm{g} / \mathrm{ml}-1)$ and $10 \%$ fetal bovine serum (FBS).

\subsection{Mitochondrial isolation}

Mitochondria were prepared from $10^{7}$ cells grown overnight in $15 \mathrm{~cm}^{2}$ dishes and isolated as described previously [56], with some modifications. Mitochondria were lysed for $30 \mathrm{~min}$ in buffer containing $250 \mathrm{mM}$ sucrose, $100 \mathrm{mM} \mathrm{KCl,} 20 \mathrm{mM}$ magnesium acetate, $10 \mathrm{mM}$ Tris- $\mathrm{HCl} \mathrm{pH} 7.5$, 0.5\% Triton X-100 and EDTA-free Complete protease inhibitor cocktail (Roche).

\subsection{RNA isolation, digestion, northern blotting and qRT-PCR}

RNA was isolated from mitochondria using the miRNeasy Mini kit (Qiagen) incorporating an on-column RNase-free DNase digestion to remove all DNA. RNA ( $5 \mu \mathrm{g}$ ) was resolved on 1.2\% agarose formaldehyde gels, then transferred to $0.45 \mu \mathrm{m}$ Hybond- $\mathrm{N}^{+}$nitrocellulose membrane (GE Lifesciences) and hybridized with biotinylated oligonucleotide probes specific to human mitochondrial mRNAs, rRNAs and tRNAs. Hybridizations were carried out overnight at $50^{\circ} \mathrm{C}$ in $5 \mathrm{x}$ SSC, $20 \mathrm{mM} \mathrm{Na} \mathrm{HPO}_{4}, 7 \%$ SDS and $100 \mu \mathrm{g} \cdot \mathrm{ml}^{-1}$ heparin, followed by washing. The signal was detected using either streptavidin-linked horseradish peroxidase or streptavidin-linked infraredlabelled antibody (diluted 1: 2000 in 3x SSC, 5\% SDS, $25 \mathrm{mM} \mathrm{Na}_{2} \mathrm{HPO}_{4}, \mathrm{pH} 7.5$ ) by enhanced chemiluminescence (GE Lifesciences) or using an Odyssey Infrared Imaging System (Li-Cor), respectively.

Complementary DNA (cDNA) was prepared using the QuantiTect Reverse Transcription Kit (Qiagen) and used as a template in the subsequent PCR that was performed using a Corbett Rotorgene 6000 using SensiMix SYBR mix (Bioline) and normalized to 18S rRNA. Isolated mitochondrial RNA $(2.5 \mu \mathrm{g})$ was incubated in the presence or absence of Terminator exonuclease (Gene Target Solutions) in Buffer B for 30 min at $42^{\circ} \mathrm{C}$ in the presence of RNaseOUT inhibitor (ThermoScientific) according to the manufacturer's instructions followed by northern blotting as described above.

\subsection{PARE and alignments}

RNA sequencing was performed on mitochondrial RNA isolated from cybrid cells using the Illumina MiSeq platform according to the PARE protocol $[6,45,46]$. 
Raw reads were processed follows: $3^{\prime}$ adaptor sequences were trimmed with cutadapt 1.9.1 (Martin, 2011) (-e 0.1 -n 2 -O 6 --quality-base=64 -match-read-wildcards -a TCGTATGCCGTCTTCTGCTTG), followed by library splitting by index sequence (5'-ATCACG$3^{\prime}$ and 5'-CGATGT-3') with fastx_barcode_splitter.pl 0.0.13 from the FASTX-Toolkit (-eol -exact), then index sequence trimming with cutadapt (-e 0.0 -n 1 -O 6 -quality-base=64). Reads that were 20 or $21 \mathrm{nt}$ in length after trimming were extracted with $a w k$ and aligned uniquely to the human mitochondrial genome (hg38) with Bowtie 1.1.2 (Ben B Langmead et al., 2008) (-yS -m 1 phred64-quals). Strand-specific 5' end profiles normalised to library size (reads per million, RPM) were generated with BEDtools 2.25.0 (Quinlan and Hall, 2010) (-d -5 -strand [-/+] -scale [1,000,000/total mapped reads]) and converted to bedGraph format for visualisation. PARE data for 143B cybrids containing wild type mtDNA was retrieved from the Gene Expression Omnibus (GEO: GSM769736) and processed as above, with the exception of specifying phred-33 rather than phred-64 at trimming and alignment steps to account for sequence quality format differences. The data generated in this study have been submitted to GEO with accession GSE85549.

\section{Results}

\subsection{The effects of $m .3243 A>G$ and $m .3302 A>G$ mutations on the processing of RNA19}

We investigated the effects of the mutations in both m.3243A>G and m.3302A $>\mathrm{G}$ cell lines to identify that the $5^{\prime}$ ends of MT-TL1 and the MT-ND1 mRNA were both dramatically reduced relative to control cells (Figs 1 and 2; Supplementary Table 1). Additionally, the ratio of MTTL1:MT-ND1 abundance in the mutants was opposite to the wild type, such that the abundance at the MT-ND1 5' end was twice that at the $5^{\prime}$ end of MT-TL1 whereas in the mutant cells the $5^{\prime}$ end of MT-TL1 is twice as abundant as MT-ND1, indicating a greater 3' end processing impairment relative to 5' end processing that was consistent with previous reports (Levinger et al., 2004; Maniura-Weber et al., 2006; Rackham et al., 2016; Rossmanith and Karwan, 1998). However, the 5'end abundance of MT-TL1, while reduced relative to the wild type cells, is not markedly different between the two mutant cell lines despite the large difference in reported $5^{\prime}$ processing efficiency for the $\mathrm{m} .3243 \mathrm{~A}>\mathrm{G}$ and $\mathrm{m} .3302 \mathrm{~A}>\mathrm{G}$ mutations indicating that both mutations lead to processing defects of MT-TL1 and consequent RNA19 accumulation (Rossmanith and Karwan, 1998).

Interestingly, we observed that the 5' end of MT-RNR2, which corresponds to the 5' end of RNA19 was approximately twice as abundant in the m.3243A $>\mathrm{G}$ cells compared to both wild type and m.3302A > G cells (Supplementary Table 1), although the significance of this is unclear. In addition to an overall reduction in abundance compared to the wild type cells, the reduction in MT-ND1 5' end abundance relative to MT-TL1 5' end abundance in the mutant cells is consistent with impaired 
3' tRNA processing previously reported for these mutations (Levinger et al., 2004). This indicates that impaired 5'cleavage has downstream effects on $3^{\prime}$ processing and further establishes that 5' tRNA processing precedes $3^{\prime}$ cleavage in mitochondria as it has been identified recently in vivo (Rackham et al., 2016). We found that the 5' end of MT-TV were also considerably more abundant in the wild type cells ( $\sim 320,000$ RPM) compared to either of the mutant cells $(\sim 60,000-69,000$ RPM) suggesting that somehow the 5'end cleavage of RNA19 was affected by the mutations in the MT-TL1 (Figs 1 and 2; Supplementary Table 1). In contrast, the 5' ends of MT-TF and MT-RNR1 upstream of RNA19 were considerably more abundant in the mutant cells than in the wild type cells, indicating variable processing efficiency when there is a mutation in MT-TL1 (Fig 1; Supplementary Table 1).

\subsection{The cleavage of non-canonical sites is altered in MT-TL1 mutant cell lines}

Mitochondrial rRNAs and mRNAs are typically released from their respective precursors RNAs via the excision of interspersed tRNAs, however several junctions are not flanked by tRNAs or are flanked by antisense tRNA sequences, namely the 3' end of MT-ND6, the junction between MT-ATP8/6 and MT-CO3, the 5' end of MT-COI and the junction between MT-ND5 and MTCYB (Figs 3 and 4). In our data two of these four junctions lacked any distinctive 5' peaks, MTND5/MT-CYB and the 5' end of the COI mRNA (Fig 3A and 3B), located 3 nt upstream of the start codon. The MT-ATP8/6-MT-CO3 junction, which lacks an intervening tRNA, was not observed in the wild type or m.3302A $>\mathrm{G}$ cells, however the m.3243A $>\mathrm{G}$ cells contained a very large peak of $\sim 32,000$ RPM (Fig 4A). Since PARE requires a 5' monophosphate to capture RNAs, we performed 5'-phosphate-dependent exonuclease digestion of isolated 143B mitochondrial RNA and revealed that MT-CO3 is unaffected (Fig 4B), suggesting the absence of this residue and, consequently, its presence in the $\mathrm{m} .3243 \mathrm{~A}>\mathrm{G}$ sample. The MT-CO3 mRNA is known to co-exist within a tricistronic transcript with MT-ATP8/6, known as RNA15, which is susceptible to the exonuclease digestion, much like the other mt-RNAs indicating that the $5^{\prime}$ of MT-CO3 is uniquely protected from digestion. The processing of the MT-CO3 mRNA from the bicistronic MT-ATP8/6 is currently poorly understood. It is possible that the FASTKD5 protein may play a role in this site since knockdown of this protein results in an increased accumulation of the RNA15 transcript along with other non-canonical junctions (Antonicka and Shoubridge, 2015). It is interesting to note that the m.3243A $>\mathrm{G}$ cells are the only ones identified to date to contain a distinctive $5^{\prime}$ peak at the MTATP8/6-MT-CO3 junction suggesting that this mutation may have downstream effects on the secondary structure of this transcript. 
A peak 34 or $35 \mathrm{nt}$ downstream of the $3^{\prime}$ end of the MT-ND6 open reading frame (ORF), as would be expected for the 3' UTR was not observed. However, small but distinctive peaks were observed in all three samples at position 13,647, which, if taken as the ND6 UTR 3' processing junction implies a $3^{\prime}$ terminal nucleotide at position 13,648, $501 \mathrm{nt}$ downstream of the MT-ND6 ORF 3' end consistent with the region described by (Slomovic et al., 2005) (Fig 4C). The 3' ends of several of these PARE tags terminated with non-templated adenosine residues, however MT-ND6 is not known to be polyadenylated suggesting that these nucleotides may be degradation-related additions. The processing of the MT-ND6 ORF 3' end appears decreased in both mutant cell lines indicating potential downstream effects of these mutations on the expression or processing of the ND6 transcript.

\subsection{Mutations in MT-TL1 lead to altered mitochondrial RNA metabolism}

Next we investigated the presence of $5^{\prime}$ peaks across the mitochondrial transcriptomes in wild-type and mutant cell lines to determine if there were any unique and non-canonical sites that are present or downstream consequences of the mutations in the MT-TL1 gene. We observed multiple large peaks located within the coding regions of several mRNAs, many of which were unique to the m.3243A>G cell line (Table 1). In this cell line MT-COI contains a peak of $\sim 38,000$ RPM at position 6,534, surrounded by four smaller peaks of $\sim 1,400-2,700$ RPM, in addition to a sixth peak $\sim 200 \mathrm{nt}$ downstream (Fig 3B). There was also a single peak of $\sim 1,500$ RPM within the MT-ND4 ORF at position 11,567 (Fig 5A) and another similarly sized peak at position 2,585 within MT-RNR1 (Fig 2) observed only in the m.3243A > G cells. These may be internal cleavage sites that are a consequence of unstable mRNAs that are downstream consequence of the $\mathrm{m} .3243 \mathrm{~A}>\mathrm{G}$ mutation. Mitochondrial protein synthesis in the m.3243A $>\mathrm{G}$ cell line is entirely lost as a result and the increased degradation of mitochondrial RNAs may be a consequence of impaired translation of all 13 mitochondria encoded polypeptides (Supplementary Fig 1). Although m.3302A>G causes impaired processing of RNA19 we do not observe an overall defect in mitochondrial translation (Supplementary Fig 1) and this may explain the lack of internal cleavage sites in mitochondrial RNAs from these cells.

We observed several instances of distinctive peaks that appear in all three samples, but which do not correspond to any known transcript ends. For example, a cluster of peaks was observed on the light strand antisense to MT-ND2 (Fig 5B), the largest and most upstream of which was located at position 5,292, and was much more abundant in the wild-type compared to mutant cell lines. Additionally, a second cluster of peaks with similar characteristics with a maximum abundance up to 18,000 RPM was observed on the light strand, in a region proximal to the MT- 
ATP8/6-MT-CO3 junction region on the heavy strand (Fig 6A), which corresponds with a previously identified region of predicted RNA secondary structure (Mercer et al., 2011). A third peak of $\sim 1,000-2,300$ RPM was observed in all samples within the MT-CO2 coding region (Fig $6 B$ ) and although its abundance was only slightly increased in the m.3243A>G mutant, relative to the $5^{\prime}$ MT-CO2 peak the ratio of internal to $5^{\prime}$ end abundance was increased in both mutants by a factor of $\sim 3-6$ (Table 1, Supplementary Table 1). These cleavage sites may be a product of RNA processing enzymes some of which can be promiscuous and cleave secondary structures within transcripts that resemble tRNAs as observed before (Rackham et al., 2011). The mutations in MTTL1 contribute to an overall defect in the hierarchy of RNA processing and may contribute to the changes in cleavage at these secondary sites.

\section{Discussion}

We investigated the effect of two mutations in MT-TL1 in the mitochondrial transcriptome by PARE. RNA19 is a mtRNA processing intermediate comprised of the complete MT-RNR2, MTTL1 and MT-ND1 sequences as a single contiguous molecule. Both mutations investigated are known to impair the processing of MT-TL1 and lead to the accumulation of RNA19 (Bindoff et al., 1993; King et al., 1992; Maniura-Weber et al., 2006), although the 5' processing defect has been reported to be more severe for the m.3243A>G mutation (Levinger et al., 2004). Our data directly sampled the 5' ends of MT-TL1 and MT-ND1 and showed reduced abundance at both junctions relative to wild type, indicative of impaired RNA19 processing as the primary defect, however there was little difference in $5^{\prime}$ MT-TL1 abundance between the two mutants despite a report that the m.3243A $>$ G mutation can also impair 5' processing (Rossmanith and Karwan, 1998). This suggests that, at least with this tRNA, the efficiency of processing of $5^{\prime}$ and $3^{\prime}$ ends is interdependent.

The 5' and 3' ends of mitochondrial RNAs are typically released from their polycistronic precursors via RNA processing enzymes acting on the tRNAs flanking the gene (reviewed in (Rackham et al., 2012)), however a number of exceptions exist: the 5' ends of MT-CO1, MT-CO3 and MT-CYB, and the 3' end of ND6. The 5' end of MT-COI is flanked by the antisense of MTTY; the $5^{\prime}$ of MT-CYB is likewise flanked by an antisense tRNA, MT-TE, but this tRNA forms a portion of the MT-ND5 3' UTR and is not processed by the canonical machinery (Sanchez et al., 2011); and the remaining two non-canonical junctions are located between MT-ATP8/6 and MT$\mathrm{CO} 3$ and the 3' end of MT-ND6, lack tRNAs entirely and have been reported to be affected by the FASTK and FASTKD5 proteins (Antonicka and Shoubridge, 2015; Jourdain et al., 2015). In our datasets, the 5' ends of MT-CO1 and MT-CYB were not detected or of very low abundance and were not appreciably different in appearance to the surrounding background. Furthermore, the MT- 
ATP8/6-MT-CO3 junction was observed as a single large peak at the 5' nucleotide of the MT-CO3 mRNA in the m.3243A>G sample only. Since PARE relies on the presence of a free 5' monophosphate to capture RNAs, the alignment of tags at the 5' end of MT-CO3 in the m.3243A>G cybrid only suggested that the $5^{\prime}$ end of this mRNA is lacking or obscured. Indeed, when isolated wild type 143B mitochondrial RNA is incubated with a 5'-phosphate-dependent exonuclease the MT-CO3 mRNA but not MT-ATP8/6 and MT-CO2, is resistant to digestion, suggesting it lacks the requisite monophosphate at its $5^{\prime}$ end unlike the other mRNAs. An alternate possibility is that the formation of an RNA secondary structure, as has been previously predicted (Mercer et al., 2011), may preclude the capture of the terminal region of this mRNA, although these possibilities are not mutually exclusive.

The location of the 3' end of MT-ND6 has been reported at both 500 nt (Slomovic et al., 2005) and 33/34 nt 3' UTRs (Mercer et al., 2011; Rackham et al., 2011). The identification of the $3^{\prime}$ junctions with this type of data is possible because of the compact, polycistronic nature of the mtRNA precursors where each $3^{\prime}$ cleavage event also creates a downstream RNA with a free 5' monophosphate. In our data here, an accumulation of tags with non-template $3^{\prime}$ adenosines within the region described by (Slomovic et al., 2005) was observed. This might indicate that the predominant 3' end of MT-ND6 occurs after 500 nt 3' UTR or, since PARE does not identify 3' ends directly, that this peak result from a long-lived, downstream degradation intermediate in the discarded light strand non-coding RNA. Since MT-ND6 is the only mitochondrial mRNA that lacks a poly(A) tail the origin of these adenosine extensions may originate with the mtPAP and the degradation machinery. Indeed, such a potential mechanism for the processing of the $3^{\prime}$ end of MTND6 that involves the degradation machinery and the FASTK protein bound to the MT-ND6 ORF and 3' UTR has been proposed (Jourdain et al., 2015).

The peaks observed within MT-ND4 and MT-RNR2 in the m.3243A>G sample do not correspond to any specific features, and their genesis is unknown. The cluster of peaks within MT$\mathrm{CO} 1$, however, resides in a region predicted to harbour RNA secondary structure and within which a large central PARE peak in HEK cells is observed (Mercer et al., 2011). The significance of the RNA structure and cleavage within this region is unclear, but its specific appearance in the m.3243A $>$ G sample suggests that changes in mitochondrial function resulting from the tRNA mutation may have changed its relative abundance and allowed its detection.

The other unidentified peaks are consistently observed in all three samples, although their origin and function are unclear. RNA structures have been predicted and cleavage detected previously (Mercer et al., 2011) within the genomic regions where peaks were observed in our data on the light strand antisense to MT-ND2 and MT-CO3. Given their staggered appearance and 
location within non-coding genomic regions these peaks may indicate protein-binding sites, particularly those involved in the processing and degradation of the light strand transcript. The other distinctive feature observed in all samples was a central peak within the MT-CO2 coding region, which was relatively abundant compared to the MT-CO2 5' end. Additionally, whilst the 5'end abundance of MT-CO2 was reduced in both mutants the abundance of the internal peak was relatively stable or even slightly increased, suggesting that MT-CO2 5' processing efficiency does not directly affect the abundance of the internal peak.

The numerous additional alternations in RNA cleavage beyond the mutated MT-TL1 indicate that there is some form of feedback causing uncoordinated RNA processing in these cells. The mechanism behind this observation is not known but it may result from the altered levels or activity of RNA processing enzymes or regulators. In support of this hypothesis, we have previously observed that PTCD1, which associates with ELAC2 and modulates processing of the 3' ends of mitochondrial tRNAs (Sanchez et al., 2011), is increased in m.3243A>G and m.3302A>G cells. Further studies will be required to understand the coordinated regulation of mitochondrial RNA processing proteins.

Taken together we show that a single mutation in a mitochondrial tRNA can lead to a processing defect by that in turn can have downstream effects on global mitochondrial processing. Details of the order of mitochondrial RNA processing are still lacking and development of new next generation technologies can significantly improve our understanding of the mechanisms of RNA cleavage in mitochondria.

Conflicts of Interest: The authors declare no conflict of interest.

Acknowledgments: This project was supported by fellowships and project grants from the National Health and Medical Research Council (APP1058442, APP1045677, APP1041582, APP1023460, APP1005030, APP1043978 to A.F. and O.R.), Australian Research Council (to A.F. and O.R.), the Cancer Council of Western Australia (to O.R.). S.J.S. was supported by a University of Western Australia Australian Postgraduate Award. 


\section{References}

Antonicka, H., Shoubridge, E.A., 2015. Mitochondrial RNA Granules Are Centers for Posttranscriptional RNA Processing and Ribosome Biogenesis. CellReports 10, 920-932. doi:10.1016/j.celrep.2015.01.030

Ben B Langmead, Trapnell, C.C., Pop, M.M., Salzberg, S.L.S., 2008. Ultrafast and memoryefficient alignment of short DNA sequences to the human genome. CORD Conference Proceedings 10, R25-R25. doi:10.1186/gb-2009-10-3-r25

Bindoff, L.A., Howell, N., Poulton, J., McCullough, D.A., Morten, K.J., Lightowlers, R.N., Turnbull, D.M., Weber, K., 1993. Abnormal RNA processing associated with a novel tRNA mutation in mitochondrial DNA. A potential disease mechanism. J Biol Chem 268, 1955919564.

Börner, G.V., Zeviani, M., Tiranti, V., Carrara, F., Hoffmann, S., Gerbitz, K.-D., Lochmuller, H., Pongratz, D., Klopstock, T., Melberg, A., Holme, E., Paabo, S., 2000. Decreased aminoacylation of mutant tRNAs in MELAS but not in MERRF patients. Hum Mol Genet 9, $467-475$.

Brzezniak, L.K., Bijata, M., Szczesny, R.J., Stepien, P.P., 2011. Involvement of human ELAC2 gene product in 3' end processing of mitochondrial tRNAs. RNA Biol 8, 616-626. doi:10.4161/rna.8.4.15393

German, M.A., Luo, S., Schroth, G., Meyers, B.C., Green, P.J., 2009. Construction of Parallel Analysis of RNA Ends (PARE) libraries for the study of cleaved miRNA targets and the RNA degradome. Nat Protoc 4, 356-362. doi:10.1038/nprot.2009.8

Goto, M., Komaki, H., Saito, T., Saito, Y., Nakagawa, E., Sugai, K., Sasaki, M., Nishino, I., Goto, Y.-I., 2014. MELAS phenotype associated with m.3302A $>\mathrm{G}$ mutation in mitochondrial tRNA(Leu(UUR)) gene. Brain Dev 36, 180-182. doi:10.1016/j.braindev.2013.03.001

Gustafsson, C.M., Falkenberg, M., Larsson, N.-G., 2016. Maintenance and Expression of Mammalian Mitochondrial DNA. Annu Rev Biochem 85, 133-160. doi:10.1146/annurevbiochem-060815-014402

Helm, M., Florentz, C., Chomyn, A., Attardi, G., 1999. Search for differences in posttranscriptional modification patterns of mitochondrial DNA-encoded wild-type and mutant human tRNALys and tRNALeu(UUR). Nucleic Acids Res 27, 756-763. doi:gkc161 [pii]

Hess, J., Parisi, M., Bennett, J., Clayton, D., 1991. Impairment of mitochondrial transcription by a point mutation associated with the MELAS subgroup of mitochondrial encephalopathies. Nature 351, 236-239.

Holzmann, J., Frank, P., Löffler, E., Bennett, K.L., Gerner, C., Rossmanith, W., 2008. RNase P without RNA: Identification and Functional Reconstitution of the Human Mitochondrial tRNA Processing Enzyme. Cell 135, 462-474. doi:10.1016/j.cell.2008.09.013

Jourdain, A.A., Koppen, M., Rodley, C.D., Maundrell, K., Gueguen, N., Reynier, P., Guaras, A.M., Enriquez, J.A., Anderson, P., Simarro, M., Martinou, J.-C., 2015. A mitochondria-specific isoform of FASTK is present in mitochondrial RNA granules and regulates gene expression and function. CellReports 10, 1110-1121. doi:10.1016/j.celrep.2015.01.063

King, M., Koga, Y., Davidson, M., Schon, E., 1992. Defects in mitochondrial protein synthesis and respiratory chain activity segregate with the tRNALeu(uur) mutation associated with mitochondrial myopathy, encephalopathy, lactic acidosis, and stroke-like episodes. Mol Cell Biol 12, 480-490.

Kirino, Y., Suzuki, T., 2005. Human mitochondrial diseases associated with tRNA wobble modification deficiency. RNA Biol 2, 41-44.

Kirino, Y., Yasukawa, T., Ohta, S., Akira, S., Ishihara, K., Watanabe, K., Suzuki, T., 2004. Codonspecific translational defect caused by a wobble modification deficiency in mutant tRNA from a human mitochondrial disease. Proc Natl Acad Sci U S A 101, 15070-15075. doi:0405173101 [pii] 
10.1073/pnas.0405173101

Levinger, L., Oestreich, I., Florentz, C., Mörl, M., 2004. A pathogenesis-associated mutation in human mitochondrial tRNALeu(UUR) leads to reduced 3 '-end processing and CCA addition. $\mathrm{J}$ Mol Biol 337, 535-544. doi:10.1016/j.jmb.2004.02.008

Levy, S., Schuster, G., 2016. Polyadenylation and degradation of RNA in the mitochondria. Biochem Soc Trans 44, 1475-1482.

Maniura-Weber, K., Helm, M., Engemann, K., Eckertz, S., Mollers, M., Schauen, M., Hayrapetyan, A., Kleist-Retzow, Von, J.-C., Lightowlers, R.N., Bindoff, L.A., Wiesner, R.J., 2006. Molecular dysfunction associated with the human mitochondrial 3302A $>\mathrm{G}$ mutation in the MTTL1 (mt-tRNALeu(UUR)) gene. Nucleic Acids Res 34, 6404-6415. doi:10.1093/nar/gk1727

Martin, M., 2011. Cutadapt removes adapter sequences from high-throughput sequencing reads. EMBnet journal.

Mercer, T.R., Neph, S., Dinger, M.E., Crawford, J., Smith, M.A., Shearwood, A.-M.J., Haugen, E., Bracken, C.P., Rackham, O., Stamatoyannopoulos, J.A., Filipovska, A., Mattick, J.S., 2011. The Human Mitochondrial Transcriptome. Cell 146, 645-658. doi:10.1016/j.cell.2011.06.051

Moraes, C.T., Ciacci, F., Bonilla, E., Jansen, C., Hirano, M., Rao, N., Lovelace, R.E., Rowland, L.P., Schon, E.A., Dimauro, S., 1993. Two novel pathogenic mitochondrial DNA mutations affecting organelle number and protein synthesis. Is the tRNA(Leu(UUR)) gene an etiologic hot spot? J Clin Invest 92, 2906-2915. doi:10.1172/JCI116913

Quinlan, A.R., Hall, I.M., 2010. BEDTools: a flexible suite of utilities for comparing genomic features. Nature Communications 26, 841-842. doi:10.1093/bioinformatics/btq033

Rackham, O., Busch, J.D., Matic, S., Siira, S.J., Kuznetsova, I., Atanassov, I., Ermer, J.A., Shearwood, A.-M.J., Richman, T.R., Stewart, J.B., Mourier, A., Milenkovic, D., Larsson, N.-G., Filipovska, A., 2016. Hierarchical RNA Processing Is Required for Mitochondrial Ribosome Assembly. CellReports 16, 1874-1890. doi:10.1016/j.celrep.2016.07.031

Rackham, O., Mercer, T.R., Filipovska, A., 2012. The human mitochondrial transcriptome and the RNA-binding proteins that regulate its expression. WIREs RNA 3, 675-695. doi:10.1002/wrna.1128

Rackham, O., Shearwood, A.-M.J., Mercer, T.R., Davies, S.M.K., Mattick, J.S., Filipovska, A., 2011. Long noncoding RNAs are generated from the mitochondrial genome and regulated by nuclear-encoded proteins. RNA 17, 2085-2093. doi:10.1261/rna.029405.111

Rossmanith, W., Karwan, R.M., 1998. Impairment of tRNA processing by point mutations in mitochondrial tRNA(Leu)(UUR) associated with mitochondrial diseases. FEBS Letts 433, 269274.

Sanchez, M.I.G.L., Mercer, T.R., Davies, S.M.K., Shearwood, A.-M.J., Nygård, K.K.A., Richman, T.R., Mattick, J.S., Rackham, O., Filipovska, A., 2011. RNA processing in human mitochondria. Cell Cycle 10, 2904-2916.

Sasarman, F., Antonicka, H., Shoubridge, E.A., 2008. The A3243G tRNALeu(UUR) MELAS mutation causes amino acid misincorporation and a combined respiratory chain assembly defect partially suppressed by overexpression of EFTu and EFG2. Hum Mol Genet 17, 3697-3707. doi:10.1093/hmg/ddn265

Slomovic, S., Laufer, D., Geiger, D., Schuster, G., 2005. Polyadenylation and Degradation of Human Mitochondrial RNA: the Prokaryotic Past Leaves Its Mark. Mol Cell Biol 25, 64276435. doi:25/15/6427 [pii]

10.1128/MCB.25.15.6427-6435.2005

van den Bosch, B.J.C., de Coo, I.F.M., Hendrickx, A.T.M., Busch, H.F.M., de Jong, G., Scholte, H.R., Smeets, H.J.M., 2004. Increased risk for cardiorespiratory failure associated with the A3302G mutation in the mitochondrial DNA encoded tRNALeu(UUR) gene. Neuromuscul Disord 14, 683-688. doi:10.1016/j.nmd.2004.06.004

Wittenhagen, L., Kelley, S., 2002. Dimerization of a pathogenic human mitochondrial tRNA. Nat 
Struct Biol 9, 586-590. 


\section{Figure Legends}

\section{Figure 1. Heatmap of canonical 5' abundance.}

Heatmap of the normalized PARE abundance at canonical $5^{\prime} \mathrm{mtRNA}$ end positions in wild type, m.3243A $>\mathrm{G}$ and m.3302A $>\mathrm{G}$ cells (first panel) and the relative change compared to wild type and the other mutant cells (second panel). The histogram shows the relative distribution of $5^{\prime}$ ends of mt-tRNAs. Positions where PARE peaks were abundant across two consecutive nucleotides are labelled numerically.

Figure 2. The effects of $m .3243 \mathrm{~A}>\mathrm{G}$ and $\mathrm{m} .3302 \mathrm{~A}>\mathrm{G}$ mutations on RNA19 processing. PARE profile across the RNA19 region (n.p. 1,546-4,348), displayed in reads per million (RPM) on a three-way split scale. The areas highlighted in blue and red indicate junctions formed by tRNA 5' and 3' processing, respectively. The area highlighted in green shows an unidentified peak observed only in the m.3243A $>\mathrm{G}$ cybrid. The peaks highlighted in purple are only observed in the wild type cells and display characteristics of degradation products, such as a stepped appearance and nontemplate polyadenylated PARE tags.

Figure 3. The cleavage of non-canonical sites is altered in MT-TL1 mutant cell lines. (A) PARE profile across the MT-ND5/MT-CYB junction (n.p. 13,548-16,049). The area highlighted in green displays the $5^{\prime}$ terminal mononucleotide of MT-CYB and its absence in all samples.

(B) PARE profile across the MT-CO1 region (n.p. 5,427-7,628). The areas highlighted in green show the cluster of internal peaks and downstream peak observed only in the m.3243A>G cells. The region highlighted in purple shows the absence of a peak at the $5^{\prime}$ terminal mononucleotide of MT-CO1 in all samples. The area highlighted in red shows the increased abundance of 3' processing MT-TW products in the mutant cells, at a junction that lacks a proximal downstream gene. The area highlighted in blue shows the $5^{\prime}$ end of mt-tRNAs.

\section{Figure 4. Mitochondrial RNAs with unusual 5' or 3' termini.}

(A) PARE profile across the MT-ATP8/6-MT-CO3 junction (n.p. 8,165-10,166). The region highlighted in purple displays the 5' terminal mononucleotide of MT-CO3 and a substantial peak that is only observed in the m.3243A $>\mathrm{G}$ cells. The area highlighted in blue shows the $5^{\prime}$ end and the area in red shows the $3^{\prime}$ end of mt-tRNAs. 
(B) Terminator exonuclease was incubated with mtRNA for 15 or $30 \mathrm{~min}$ at $42^{\circ} \mathrm{C}$ to show that the canonical 5' terminal mononucleotide of MT-CO3 is resistant to digestion compared to MTATP8/6 and MT-CO2.

(C) PARE profile across the MT-ND6 region (n.p. 12,258-14,759). The light strand annotation displays the ND6 coding region, and the long and short ND6 3' UTR. The peaks highlighted in purple are located in the approximate region described by long UTR, and the PARE tags possess 3' non-template adenosines. The area highlighted in blue shows the $5^{\prime}$ end and the area in red shows the $3^{\prime}$ end of mt-tRNAs.

\section{Figure 5. Mutations in MT-TL1 lead to altered mitochondrial RNA metabolism.}

(A) PARE profile across the MT-ND4L/4 region (n.p. 10,351-12,352). The peak highlighted in green is observed only in the m.3243A>G cells; the peaks highlighted in blue and red are junctions processed by RNase P and ELAC2, respectively.

(B) PARE profile across the MT-ND2 - WANCY region (n.p. 5,050-6,050). The region highlighted in green shows the cluster of peaks observed on the light strand antisense to the $3^{\prime}$ end of MT-ND2; the peaks highlighted in blue and red are junctions processed by RNase P and ELAC2, respectively.

Figure 6. Consequences of mutations in MT-TL1 on mitochondrial RNAs.

(A) PARE profile across the antisense MT-ATP8/6-MT-CO3 junction (n.p. 8,700-9,700). The peaks highlighted in green are observed in all samples and do not correspond with any known transcript end.

(B) PARE profile across the MT-CO2 region (n.p. 7,430-8,430). The peak highlighted in green does not correspond to any known transcript end and is observed in all samples; the peaks highlighted in blue and red are junctions processed by RNase P and ELAC2, respectively. 


\begin{tabular}{|c|c|c|c|c|c|c|c|c|}
\hline \multirow[b]{2}{*}{ Strand } & \multirow[b]{2}{*}{ ID } & \multirow[b]{2}{*}{ Position } & \multicolumn{3}{|c|}{ Reads per million (RPM) } & \multicolumn{3}{|c|}{$\log _{2}$ FoldChange (RPM+1) } \\
\hline & & & WT & A3243G & A3302G & A3243G/WT & A3302G/WT & A3243G/A3302G \\
\hline \multirow[t]{9}{*}{ Heavy } & 16S_internal & 2,585 & 0.00 & $1,047.82$ & 5.04 & 10.03 & 2.59 & 7.44 \\
\hline & CO1_internal_1 & 6,514 & 0.00 & $1,688.16$ & 0.00 & 10.72 & 0.00 & 10.72 \\
\hline & CO1_internal_2 & 6,528 & 0.00 & $1,397.10$ & 1.01 & 10.45 & 1.01 & 9.44 \\
\hline & CO1_internal_3 & 6,531 & 0.00 & $1,891.90$ & 2.02 & 10.89 & 1.59 & 9.29 \\
\hline & CO1_internal_4 & 6,534 & 0.00 & $37,983.50$ & 3.02 & 15.21 & 2.01 & 13.20 \\
\hline & CO1_internal_5 & 6,536 & 0.00 & $2,677.77$ & 3.02 & 11.39 & 2.01 & 9.38 \\
\hline & CO1_internal_6 & 6,766 & 0.00 & $1,513.52$ & 1.01 & 10.56 & 1.01 & 9.56 \\
\hline & $\mathrm{CO} 2 \_$internal & 7,998 & 967.96 & $2,299.39$ & $1,187.92$ & 1.25 & 0.30 & 0.95 \\
\hline & ND4_internal & 11,567 & 0.00 & $1,513.52$ & 2.02 & 10.56 & 1.59 & 8.97 \\
\hline \multirow[t]{2}{*}{ Light } & CO3_antisense & 9,287 & $17,829.30$ & $5,035.36$ & $2,371.81$ & -1.82 & -2.91 & 1.09 \\
\hline & ND2_antisense & 5,292 & $4,496.35$ & 291.06 & 122.92 & -3.94 & -5.18 & 1.24 \\
\hline
\end{tabular}

Table 1: PARE tag abundance at non-canonical positions across the mitochondrial genome.

Normalised abundances (reads per million; RPM) and relative changes ( $\log _{2}$ fold change of RPM values plus a pseudocount of 1) of PARE tags at non-canonical positions. 

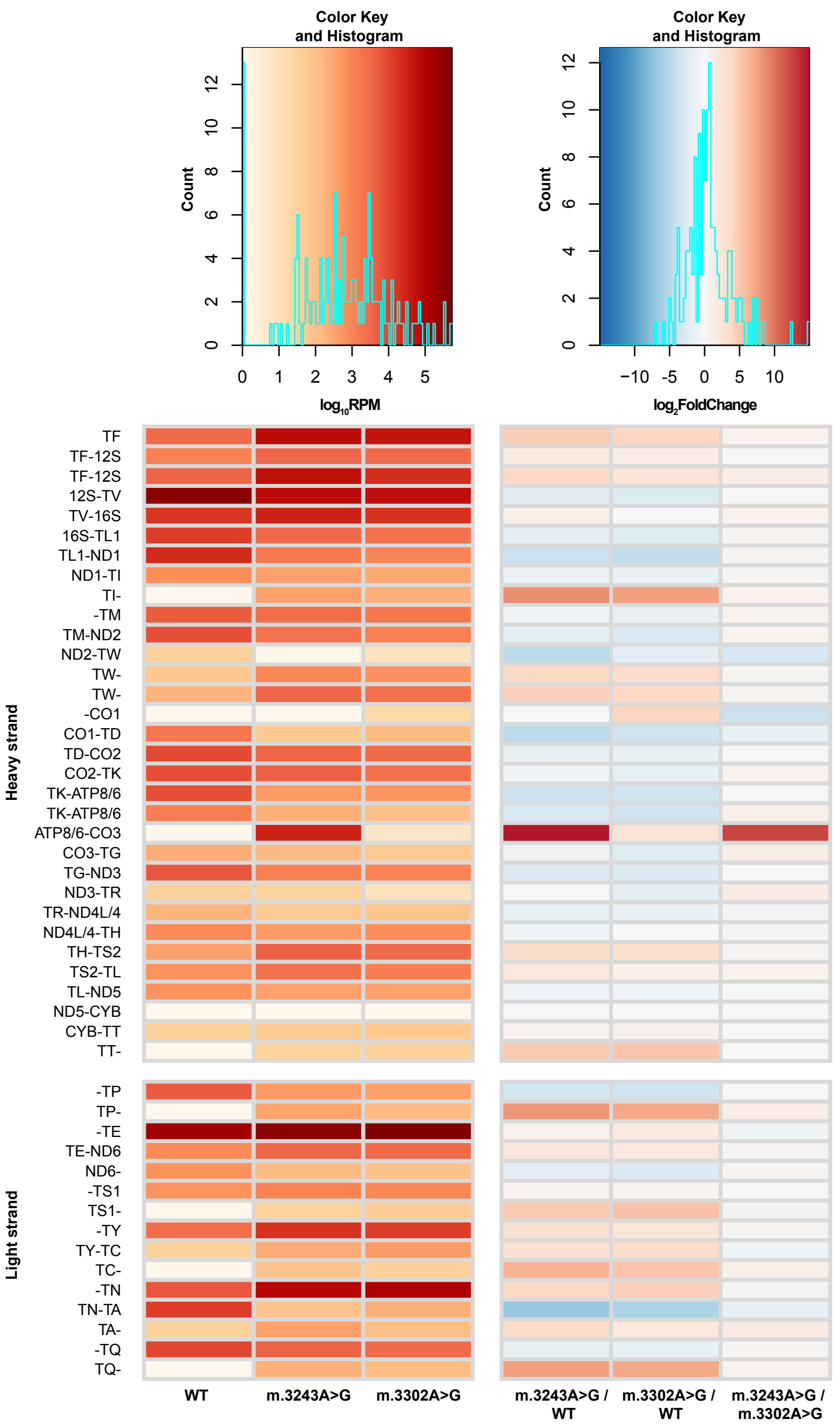


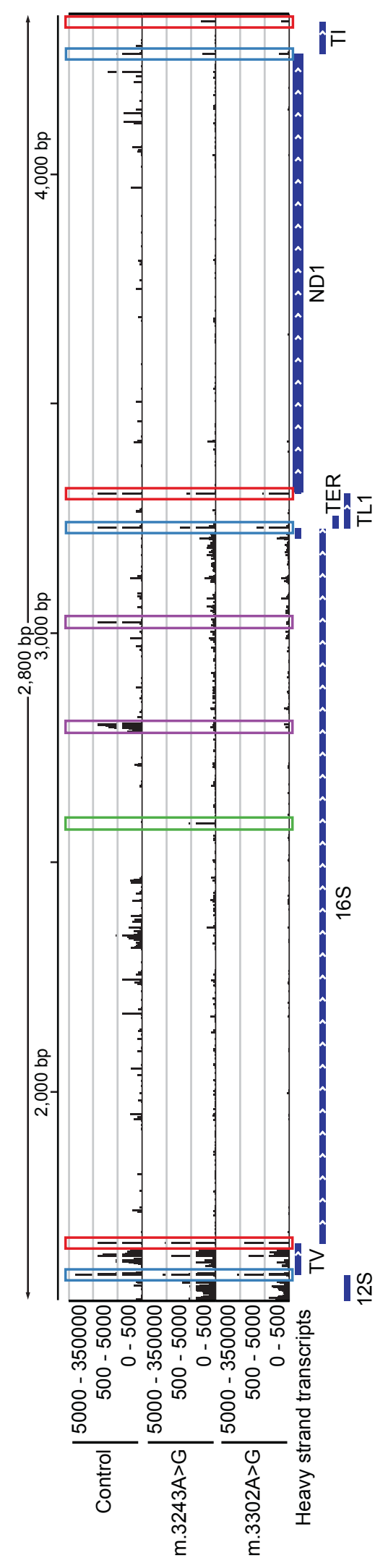


Figure 3

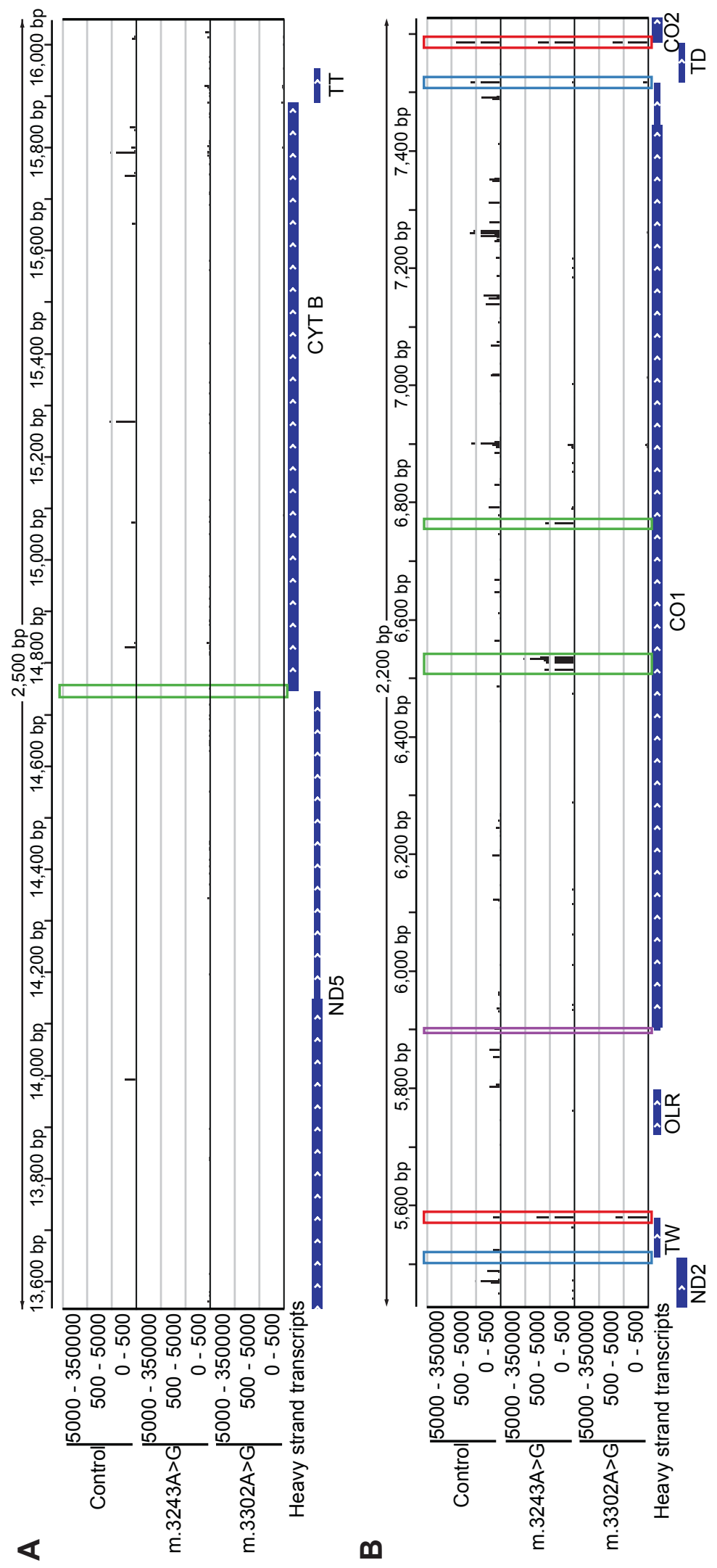


Figure 4

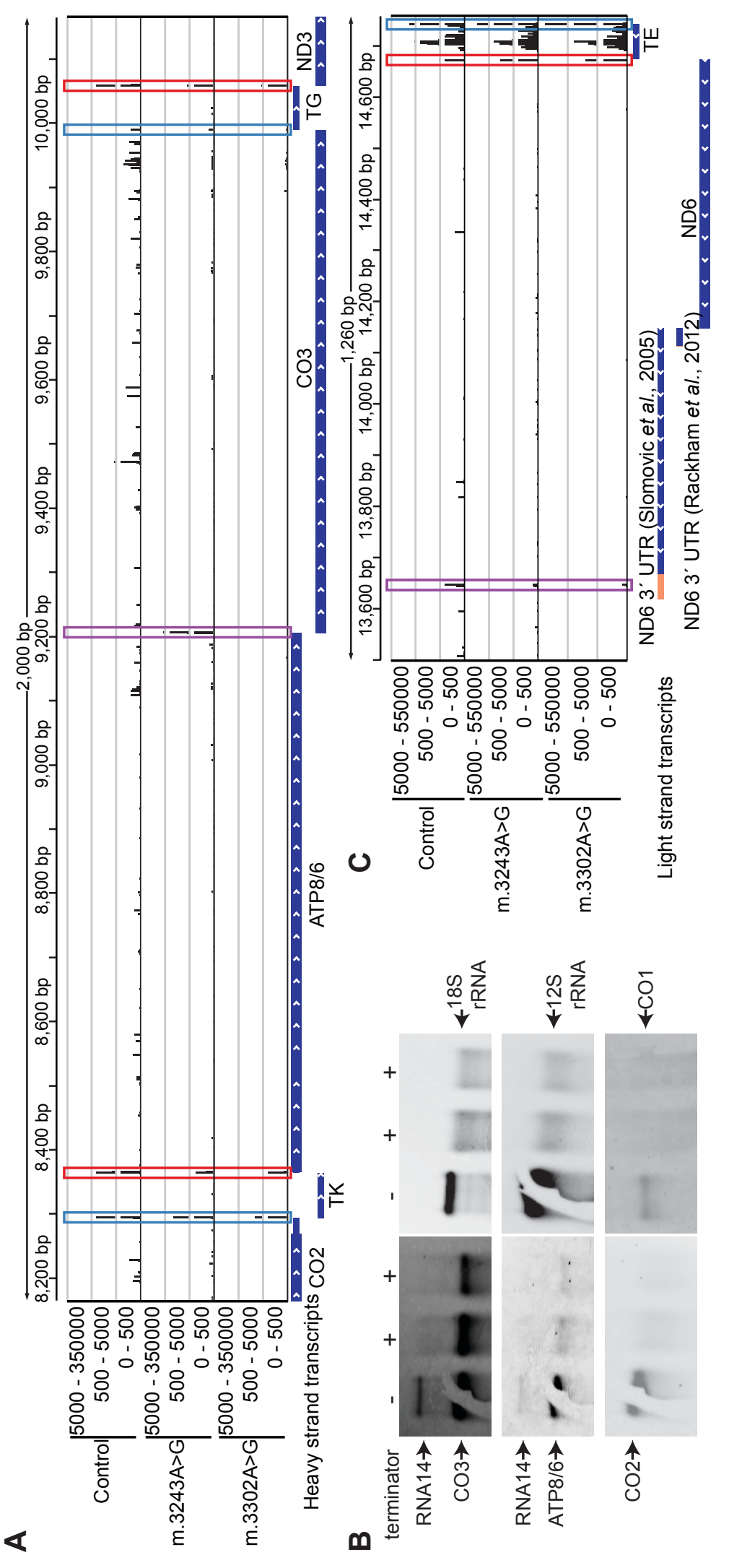




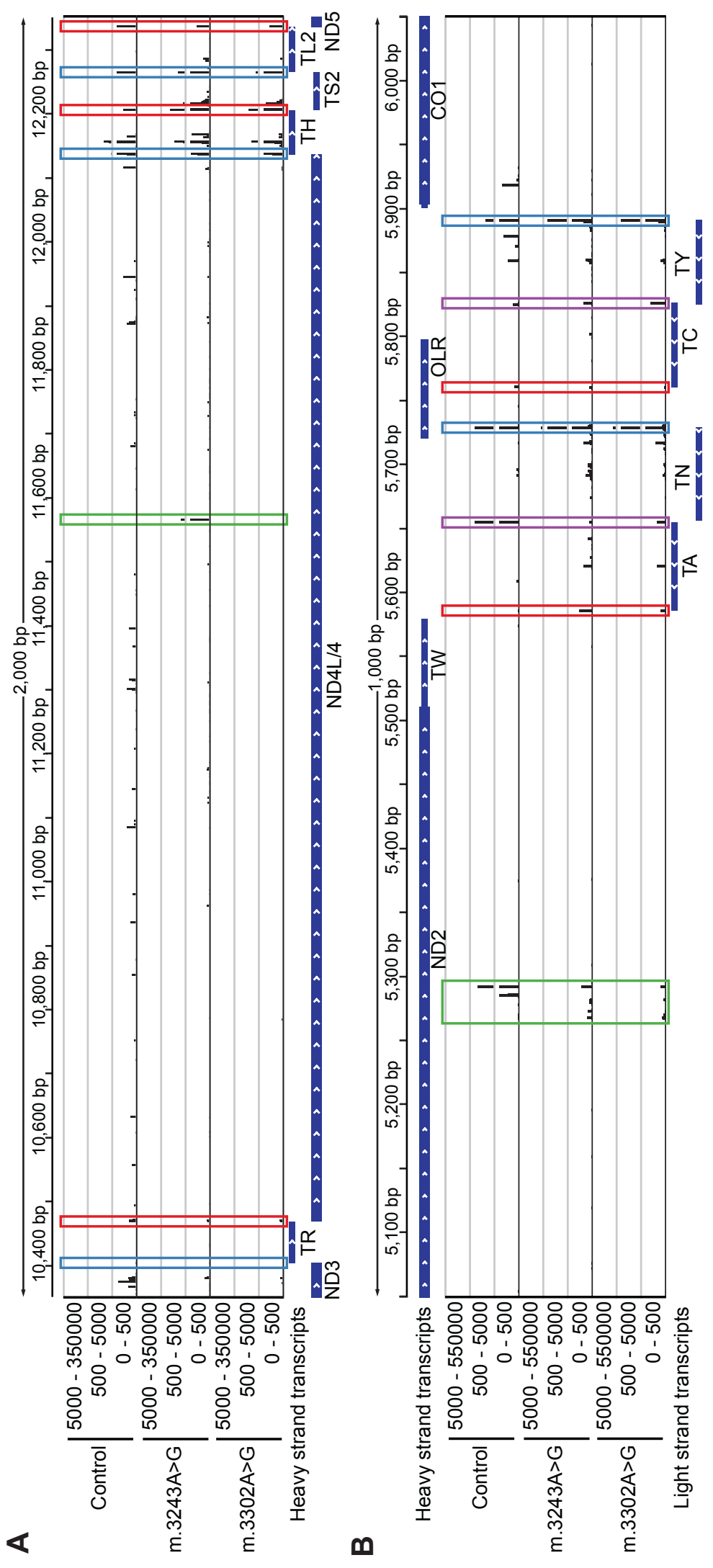




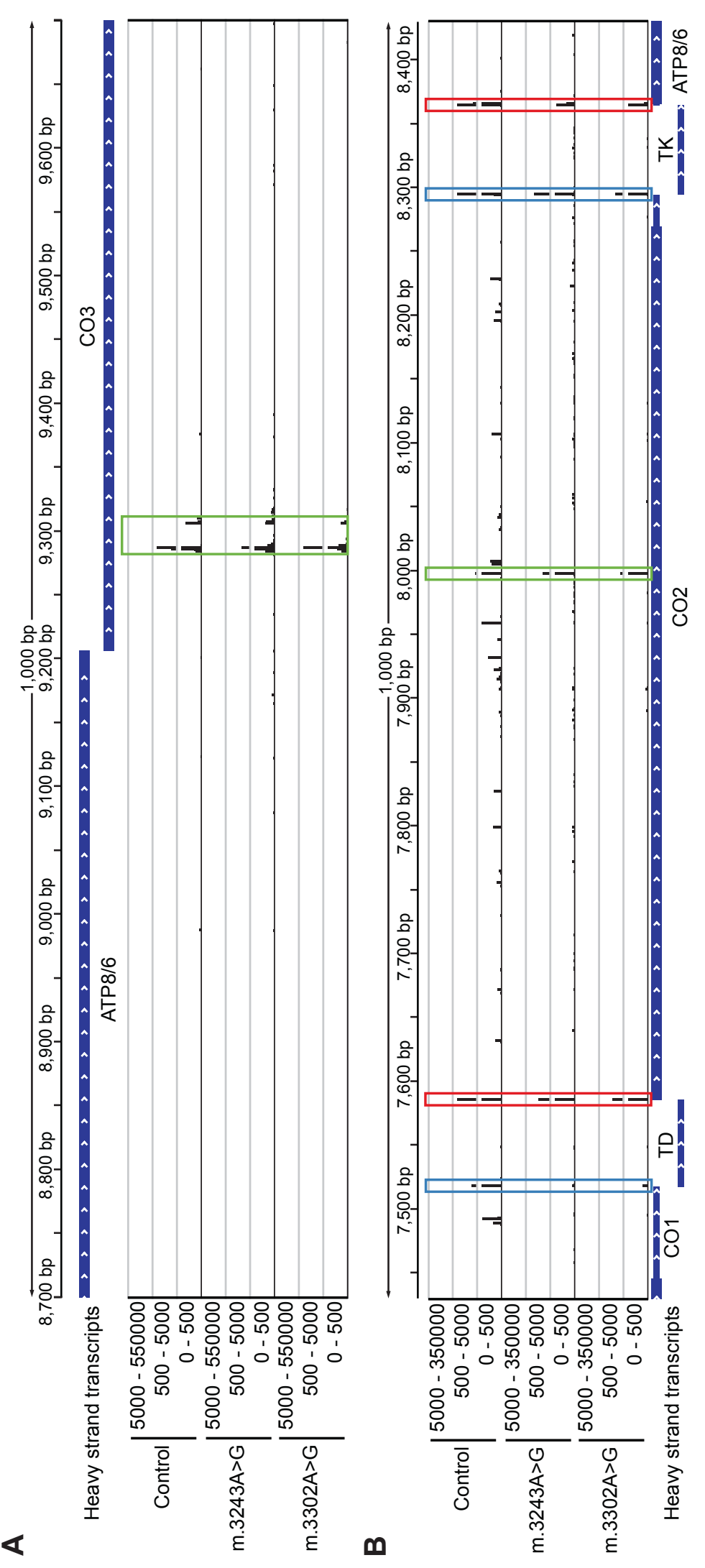




\begin{tabular}{|c|c|c|c|c|c|c|c|c|}
\hline \multirow[b]{2}{*}{ Strand } & \multirow[b]{2}{*}{ ID } & \multirow[b]{2}{*}{ Position } & \multicolumn{3}{|c|}{ Reads per million (RPM) } & \multicolumn{3}{|c|}{$\log _{2}$ FoldChange (RPM+1) } \\
\hline & & & WT & A3243G & A3302G & A3243G/WT & A3302G/WT & A3243G/A3302G \\
\hline \multirow[t]{9}{*}{ Heavy } & 16S_internal & 2,585 & 0.00 & $1,047.82$ & 5.04 & 10.03 & 2.59 & 7.44 \\
\hline & CO1_internal_1 & 6,514 & 0.00 & $1,688.16$ & 0.00 & 10.72 & 0.00 & 10.72 \\
\hline & CO1_internal_2 & 6,528 & 0.00 & $1,397.10$ & 1.01 & 10.45 & 1.01 & 9.44 \\
\hline & CO1_internal_3 & 6,531 & 0.00 & $1,891.90$ & 2.02 & 10.89 & 1.59 & 9.29 \\
\hline & CO1_internal_4 & 6,534 & 0.00 & $37,983.50$ & 3.02 & 15.21 & 2.01 & 13.20 \\
\hline & CO1_internal_5 & 6,536 & 0.00 & $2,677.77$ & 3.02 & 11.39 & 2.01 & 9.38 \\
\hline & CO1_internal_6 & 6,766 & 0.00 & $1,513.52$ & 1.01 & 10.56 & 1.01 & 9.56 \\
\hline & CO2_internal & 7,998 & 967.96 & $2,299.39$ & $1,187.92$ & 1.25 & 0.30 & 0.95 \\
\hline & ND4_internal & 11,567 & 0.00 & $1,513.52$ & 2.02 & 10.56 & 1.59 & 8.97 \\
\hline \multirow[t]{2}{*}{ Light } & CO3_antisense & 9,287 & $17,829.30$ & $5,035.36$ & $2,371.81$ & -1.82 & -2.91 & 1.09 \\
\hline & ND2_antisense & 5,292 & $4,496.35$ & 291.06 & 122.92 & -3.94 & -5.18 & 1.24 \\
\hline
\end{tabular}

Table 1: PARE tag abundance at non-canonical positions across the mitochondrial genome.

Normalised abundances (reads per million; RPM) and relative changes ( $\log _{2}$ fold change of RPM values plus a pseudocount of 1) of PARE tags at non-canonical positions. 
Supplementary Files
Click here to download Supplementary Files: Supplementary Information.docx

Supplementary Files
Click here to download Supplementary Files: Supplementary Information.docx

Fion.

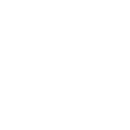

(1)

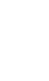

$\sqrt{2}$ (1)

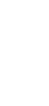
(1) (1)

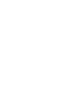

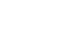
(1) (1) (1) (1) . . . . . . . . . . . . . . . . 